This is an accepted manuscript of an article published by Taylor \& Francis in International Journal of General Systems on 3 Oct 2019, available online: https://doi.org/10.1080/03081079.2019.1668386

\title{
Orness for real m-dimensional interval-valued OWA operators and its application to determine a good partition
}

\author{
L. De Miguel ${ }^{\mathrm{a}}$, D. Paternain ${ }^{\mathrm{a}}$, I. Lizasoain ${ }^{\mathrm{b}}$, G. Ochoa ${ }^{\mathrm{b}}$ and H. Bustince ${ }^{\mathrm{a}}$ \\ ${ }^{a}$ Department of Statistic, Computer Science and Mathematics and Institute of Smart Cities, \\ Public University of Navarre, Campus Arrosadia s/n 31006 Pamplona, Spain \\ b Department of Statistic, Computer Science and Mathematics and Institute for Adavanced \\ Materials, Public University of Navarre, Campus Arrosadia s/n 31006 Pamplona, Spain
}

\author{
ARTICLE HISTORY \\ Compiled May 7, 2020
}

\begin{abstract}
Ordered Weighted Averaging (OWA) operators are a profusely applied class of averaging aggregation functions, i.e., operators that always yield a value between the minimum and the maximum of the inputs. The orness measure was introduced to classify the behavior of the OWA operators depending on the weight vectors. Defining a suitable orness measure is an arduous task when we deal with OWA operators defined over more intricate spaces, such us intervals or lattices. In this work we propose a suitable definition for the orness measure to classify OWA operators defined on the set of $m$ dimensional intervals taking real values in $[0,1]$. The orness measure is applied to decide which is the best partition of a continuous range that should be divided into four linguistic labels. This example shows the good behavior of the proposed orness measure.
\end{abstract}

\section{KEYWORDS}

OWA operator; interval-valued fuzzy sets ; orness measure; partition.

\section{Introduction}

In data mining, data preprocessing is a fundamental step necessary to improve the quality and representation of data to be processed (García, Luengo, and Herrera 2015; Astorino et al. 2011). One of the preprocessing tasks is discretization, that consists in partitioning a continuous range (domain of a variable) into a set of non-overlapping intervals (Liu et al. 2002). There is not a standard method to fulfill this process (Choi and Moon 2004; Lavangnananda and Chattanachot 2017). The main reason is that the best discretization scheme strongly depends on the context of the considered application. For instance, in some situations it is advisable to take a partition with an equally wide range for all the classes while ranges with different widths are preferred in other cases. In this paper, we consider the lattice of $m$-intervals in order to ease the process of discretization in data prepocessing. For this purpose, we generalize the notion of orness measure for OWA operators which are defined over the lattice of $m$-dimensional intervals in the unit interval.

OWA operators were introduced by Yager in (Yager 1988). Since then, many authors 
have studied the generalization of OWA operator to other spaces (De Miguel et al. 2017; Wang and Xu 2016; Wei et al. 2016; Merigó and Casanovas 2010; Yager 2009; Jin, Mesiar, and Yager 2019; Paternain et al. 2019). The extension of OWA operators to interval-valued fuzzy sets has been studied in (Bustince et al. 2013) and in (De Miguel et al. 2016) by defining a linear order between the real intervals. In a complementary way, OWA operators are generalized in (Lizasoain and Moreno 2013; Mesiar et al. $2018)$ to fuzzy sets that take values on a complete lattice $\left(L, \leq_{L}\right)$, even in the case where the order $\leq_{L}$ is not linear on $L$. A particular case is the lattice $\mathbb{I}_{m}$ comprising all the real $m$ dimensional intervals $\left[a_{1}, \ldots, a_{m}\right]$ with $0 \leq a_{1} \leq \cdots \leq a_{m} \leq 1$ with the partial order $\leq$ given by

$$
\left[a_{1}, \ldots, a_{m}\right] \leq\left[c_{1}, \ldots, c_{m}\right] \text { if and only if } a_{1} \leq c_{1}, \ldots, a_{m} \leq c_{m}
$$

In this work, we investigate the lattice $\mathbb{I}_{m}$ as a mathematical model to deal with the different partitions of a continuous and bounded range. This lattice is particularly simple and it gives an elemental solution to the task of associating each class with its corresponding range. In particular, in this paper we introduce the notion of orness measure for OWA operators defined over $\mathbb{I}_{m}$. We include an example in which the orness measure is applied to choose the best weight vector to fusion three different partitions corresponding to four linguistic labels. The advantage of the proposed orness measure is that it shows a good behavior, i.e., the higher the elements of the weight vector are, the higher the orness measure is.

The structure of the paper is as follows. In Section 2, we introduce some preliminaries studies of OWA operators and orness measures. In Section 3, we deal with OWA operators defined over the set of m-dimensional intervals introducing the definition of the orness measure. We show the applicability of the proposed notion to decide an appropriate partition of a continuous and bounded range in Section 4. We finish with some conclusions and marking some future lines of research.

\section{Preliminaries}

Throughout this paper $\left(L, \leq_{L}\right)$ denotes a complete lattice, i.e., a partially ordered set in which all subsets have both a supremum and an infimum. $0_{L}$ and $1_{L}$ respectively stand for the least and the greatest elements of the lattice $L$. For more information, see (Birkhoff 1967; Davey and Priestley 1990).

Recall that an $n$-ary aggregation function defined over the lattice $L$ (see (Komorníková and Mesiar 2011)) is a function $M: L^{n} \rightarrow L$ such that:

(1) $M\left(a_{1}, \ldots, a_{n}\right) \leq_{L} M\left(a_{1}^{\prime}, \ldots, a_{n}^{\prime}\right)$ whenever $a_{i} \leq_{L} a_{i}^{\prime}$ for $1 \leq i \leq n$.

(2) $M\left(0_{L}, \ldots, 0_{L}\right)=0_{L}$ and $M\left(1_{L}, \ldots, 1_{L}\right)=1_{L}$.

It is said to be idempotent if $M(a, \ldots, a)=a$ for every $a \in L$ and it is called symmetric if, for every permutation $\sigma$ of the set $\{1, \ldots, n\}, M\left(a_{1}, \ldots, a_{n}\right)=M\left(a_{\sigma(1)}, \ldots, a_{\sigma(n)}\right)$.

Definition 2.1. (De Baets and Mesiar 1999) A map $T: L \times L \rightarrow L$ is said to be a t-norm (resp. t-conorm) on $\left(L, \leq_{L}\right)$ if it is commutative, associative, increasing in each component and has a neutral element $1_{L}\left(\right.$ resp. $\left.0_{L}\right)$.

Remark 1. Although t-conorms are formally defined as binary operations, $S$ : $L \times L \rightarrow L$, the associativity property ensures that we can compute the values for any finite tuple of $n$ inputs with $n>2$. Indeed, the simplification $S\left(a_{1}, \ldots, a_{n}\right)=$ 
$\left.S\left(S\left(S\left(a_{1}, a_{2}\right), a_{3}\right), \ldots, a_{n-1}\right), a_{n}\right)$ is frequently used. Note that the order of the inputs lacks of importance since t-conorms are symmetric functions.

Definition 2.2. (Yager 1988) For each weight vector $\alpha=\left(\alpha_{1}, \ldots, \alpha_{n}\right) \in[0,1]^{n}$, with $\alpha_{1}+\ldots+\alpha_{n}=1$, the map $F_{\alpha}:[0,1]^{n} \rightarrow[0,1]$ given by

$$
F_{\alpha}\left(a_{1}, \ldots, a_{n}\right)=\alpha_{1} a_{\sigma(1)}+\ldots+\alpha_{n} a_{\sigma(n)} ; a_{\sigma(1)} \geq \ldots \geq a_{\sigma(n)}
$$

is called an Ordered Weighted Averaging (OWA) operator.

OWA operators form a family of symmetric weighted average operators situated between the AND-operator (given by the minimum) and the OR-operator (given by the maximum), which includes the arithmetic mean.

With the purpose of classifying these operators, Yager introduced in (Yager 1988, 1993) an orness measure for each OWA operator $F_{\alpha}$, which depends only on the weight vector $\alpha=\left(\alpha_{1}, \ldots, \alpha_{n}\right)$, in the following way:

$$
\operatorname{orness}\left(F_{\alpha}\right)=\frac{1}{n-1} \sum_{j=1}^{n}(n-j) \alpha_{j} .
$$

It is easy to check that the orness of each operator is a real value situated between 0 and 1 . The orness of the AND-operator, provided by the weight vector $(0, \ldots, 0,1)$, is equal to 0 and the orness of the OR-operator, provided by the weight vector $(1,0, \ldots, 0)$, is equal to 1 . In general, the orness is a measure of the proximity of each OWA operator to the OR-operator. For instance, the orness of the arithmetic mean, provided by the weight vector $(1 / n, \ldots, 1 / n)$, is equal to $1 / 2$.

Let $\left(L, \leq_{L}, T, S\right)$ denote a quadruple in which $\left(L, \leq_{L}\right)$ is a complete lattice endowed with a t-norm $T$ and a t-conorm $S$. In (Lizasoain and Moreno 2013), the notion of OWA operators is extended from the real unit interval to any arbitrary complete lattice $L$, whenever the weight vector $\alpha=\left(\alpha_{1}, \ldots, \alpha_{n}\right) \in L^{n}$ satisfies that $S\left(\alpha_{1}, \ldots, \alpha_{n}\right)=1_{L}$ and

$$
T\left(a, S\left(\alpha_{1}, \ldots, \alpha_{n}\right)\right)=S\left(T\left(a, \alpha_{1}\right), \ldots, T\left(a, \alpha_{n}\right)\right), \text { for any } a \in L .
$$

We refer to these vectors as distributive weight vectors.

Remark 2. Note that finding distributive weight vectors may not be an easy task. Fortunately, in (Lizasoain and Moreno 2013), it is proven that if $\left(L, \leq_{L}, \wedge, \vee\right)$ is a complete distributive lattice, then any weight vector $\left(\alpha_{1}, \ldots, \alpha_{n}\right) \in L^{n}$ satisfying $\alpha_{1} \vee$ $\ldots \vee \alpha_{n}=1_{L}$ is distributive.

Definition 2.3. (Lizasoain and Moreno 2013) Let $\left(L, \leq_{L}, T, S\right)$ be a quadruple. For each distributive weight vector $\alpha=\left(\alpha_{1}, \ldots, \alpha_{n}\right) \in L^{n}$, the $n$-ary $O W A$ operator $F_{\alpha}$ : $L^{n} \rightarrow L$ is defined by

$$
F_{\alpha}\left(a_{1}, \ldots, a_{n}\right)=S\left(T\left(\alpha_{1}, b_{1}\right), \ldots, T\left(\alpha_{n}, b_{n}\right)\right)
$$

where, for each $\left(a_{1}, \ldots, a_{n}\right) \in L^{n}$, the elements $b_{n} \leq_{L} \ldots \leq_{L} b_{1}$ are calculated by means of the $k$-th statistics $\left\{A_{k}: L^{n} \rightarrow L \mid 1 \leq k \leq n\right\}$ below: 
- $b_{1}=A_{1}\left(a_{1}, \ldots, a_{n}\right)=a_{1} \vee \ldots \vee a_{n} \in L$.

- $b_{2}=A_{2}\left(a_{1}, \ldots, a_{n}\right)=\left(\left(a_{1} \wedge a_{2}\right) \vee \ldots \vee\left(a_{1} \wedge a_{n}\right)\right) \vee\left(\left(a_{2} \wedge a_{3}\right) \vee \ldots \vee\left(a_{2} \wedge a_{n}\right)\right) \vee$

$\ldots \vee\left(a_{n-1} \wedge a_{n}\right) \in L$.

- $b_{k}=A_{k}\left(a_{1}, \ldots, a_{n}\right)=\bigvee\left\{a_{j_{1}} \wedge \ldots \wedge a_{j_{k}} \mid j_{1}<\ldots<j_{k} \in\{1, \ldots, n\}\right\} \in L$

- $b_{n}=A_{n}\left(a_{1}, \ldots, a_{n}\right)=a_{1} \wedge \ldots \wedge a_{n} \in L$

Remark 3. Let $\left(L, \leq_{L}, T, S\right)$ be a quadruple, $\left(a_{1}, \ldots, a_{n}\right) \in L^{n}$ and $\left(b_{1}, \ldots, b_{n}\right)$ the elements considered in Def. 2.3. The following statements hold.

(1) $a_{1} \wedge \ldots \wedge a_{n}=b_{n} \leq_{L} b_{n-1} \leq \ldots \leq_{L} b_{2} \leq_{L} b_{1}=a_{1} \vee \ldots \vee a_{n}$

(2) If the set $\left\{a_{1}, \ldots, a_{n}\right\}$ is totally ordered, then $\left(b_{1}, \ldots, b_{n}\right)$ is simply a rearrangement of $\left(a_{1}, \ldots a_{n}\right)$ with $b_{1} \geq_{L} \ldots \geq_{L} b_{n}$.

(3) $F_{\alpha}$ is an idempotent symmetric $n$-ary aggregation function lying between the operators defined by the meet and the join on $L$. In other words, $F_{\alpha}$ is an averaging operator.

(4) The $k$-th statistics $\left\{A_{k}: L^{n} \rightarrow L \mid 1 \leq k \leq n\right\}$ are particular cases of OWA operators. Indeed, for any $1 \leq k \leq n$, consider the weight vector $\left(\alpha_{1}, \ldots, \alpha_{n}\right)$, with $\alpha_{k}=1_{L}$ and $\alpha_{j}=0_{L}$ for all $j \neq k$. Then the OWA operator $F_{\alpha}$ coincides with $A_{k}$.

In 2016, Paternain et al. define a quantitative orness ${ }^{1}$ for any $n$-ary OWA operator $F_{\alpha}: L^{n} \rightarrow L$ whenever the lattice $\left(L, \leq_{L}\right)$ satisfies the condition (MFC) given by:

(MFC) For any $a, b \in L$ with $a \leq_{L} b$, there exists some maximal chain between $a$ and $b, a=a_{0}<_{L} a_{1}<_{L} \ldots<_{L} a_{l}=b$, with a finite length $l$, where the maximality means that, for any $0 \leq i \leq l-1$ there is no $c \in L$ with $a_{i}<_{L} c<_{L} a_{i+1}$.

Definition 2.4. (Paternain et al. 2016) Let $\left(L, \leq_{L}, T, S\right)$ be a quadruple, where $\left(L, \leq_{L}\right.$ ) is a complete lattice satisfying condition (MFC). For any distributive weight vector $\alpha=\left(\alpha_{1}, \ldots, \alpha_{n}\right) \in L^{n}$, consider the qualitative quantifier $Q_{\alpha}:\{0,1, \ldots, n\} \rightarrow L$ given by:

$$
\begin{aligned}
& Q_{\alpha}(0)=0_{L} \\
& Q_{\alpha}(j)=S\left(\alpha_{1}, \ldots, \alpha_{j}\right), \text { for } j=1, \ldots, n .
\end{aligned}
$$

For each $j=1, \ldots, n$, call $\mu(j)=d_{L}\left(Q_{\alpha}(j-1), Q_{\alpha}(j)\right)$, a distance defined as the length of the shortest maximal chain between $Q_{\alpha}(j-1)$ and $Q_{\alpha}(j)$.

If $\mu=\mu(1)+\ldots+\mu(n)$, then define

$$
\operatorname{orness}\left(F_{\alpha}\right)=\frac{1}{n-1} \sum_{j=1}^{n}(n-j) \frac{\mu(j)}{\mu} .
$$

In the conditions of Definition 2.4, the concept of orness $\left(F_{\alpha}\right)$ is well-defined, i.e., if $F_{\alpha}=F_{\beta}$ for some distributive weight vector $\beta \in L^{n}$, then $\operatorname{orness}\left(F_{\alpha}\right)=\operatorname{orness}\left(F_{\beta}\right)$.

\footnotetext{
${ }^{1}$ In the literature, there is also a definition of qualitative orness (Ochoa et al. 2017), but this is out of the scope of this work.
} 
Note that, since the unit interval $[0,1]$ does not satisfy the condition MFC, the orness defined in Eq. (2) is not a valid expression. However, there is a natural definition of distance $d(a, b)$ between two elements $a$ and $b$ in $[0,1]$, named $d(a, b)=|b-a|$. If this distance is placed instead of $d_{L}\left(Q_{\alpha}(j-1), Q_{\alpha}(j)\right)$ in the previous formula, Yager's orness is recovered since:

$$
d_{[0,1]}\left(Q_{\alpha}(j-1), Q_{\alpha}(j)\right)=\alpha_{j} \text { for each } j=0, \ldots, n \text {. }
$$

\section{OWA operators on the set of $m$ dimensional intervals}

Before considering the lattice $\mathbb{I}_{m}$ comprising all the $m$-dimensional intervals contained in $[0,1]$, we recall some results obtained in (De Miguel et al. 2018) about the quantitative orness of OWA operators defined over $\mathbb{L}_{m}$, where $\mathbb{L}_{m}$ denotes the $m$ dimensional intervals with bounds in a complete lattice $L$ satisfying the (MFC) condition. Let $\left(L, \leq_{L}, T, S\right)$ be a quadruple, where $\left(L, \leq_{L}\right)$ is a complete lattice satisfying the MFC condition and endowed with a t-norm $T$ and a t-conorm $S$.

We refer to the elements of $\mathbb{L}_{m}$ with boldface letters $\boldsymbol{a}=\left[a_{1}, \ldots, a_{m}\right]$ where $a_{i} \in L$ and they satisfy $a_{1} \leq_{L} \ldots \leq_{L} a_{m}$. It is worth mentioning that $\left(\mathbb{L}_{m}, \leq_{\mathbb{L}_{m}}\right)$ is also a complete lattice with the order relation $\leq_{\mathbb{L}_{m}}$ given by

$$
\boldsymbol{a} \leq_{\mathbb{L}_{m}} \boldsymbol{c} \text { if and only if } a_{i} \leq_{L} c_{i} \text { for } i=1, \ldots, m
$$

Furthermore, the map $\mathbb{T}: \mathbb{L}_{m} \times \mathbb{L}_{m} \rightarrow \mathbb{L}_{m}$ given, for any $\boldsymbol{a}, \boldsymbol{c} \in \mathbb{L}_{m}$ by

$$
\mathbb{T}(\boldsymbol{a}, \boldsymbol{c})=\left[T\left(a_{1}, c_{1}\right), \ldots, T\left(a_{m}, c_{m}\right)\right]
$$

is a representable t-norm on $\mathbb{L}_{m}$. Similarly, the map $\mathbb{S}: \mathbb{L}_{m} \times \mathbb{L}_{m} \rightarrow \mathbb{L}_{m}$ given, for any $\boldsymbol{a}, \boldsymbol{c} \in \mathbb{L}_{m}$ by

$$
\mathbb{S}(\boldsymbol{a}, \boldsymbol{c})=\left[S\left(a_{1}, c_{1}\right), \ldots, S\left(a_{m}, c_{m}\right)\right]
$$

is a representable t-conorm on $\mathbb{L}_{m}$.

It is worth mentioning that the definition of representable t-norms (t-conorms) is more general than the one considered in this paper, since different t-norms $T_{1}, \ldots T_{m}$ (tconorms $\left.S_{1}, \ldots, S_{m}\right)$ satisfying appropriate properties can be considered in each component of the m-dimensional interval. See Lizasoain and Ochoa 2014, for more information on representable t-norms (t-conorms) on $L_{m}$. Similarly, pseudo-representable or non-representable t-norms (t-conorms) can be considered, see Deschrijver and Cornelis 2007; Deschrijver 2008. In general, we believe there will be no big differences if some other t-norms and t-conorms are considered in the paper whenever the weight vectors are distributive and they generate $m$-dimensional intervals. However, for the sake of simplicity in the notation, we only consider in this work the above notions of representable t-norms and t-conorms.

In (De Miguel et al. 2018), it is shown that the lattice $\left(\mathbb{L}_{m}, \leq_{\mathbb{L}_{m}}\right)$ satisfies the (MFC) property when $\left(L, \leq_{L}\right)$ does. This means that a suitable definition of the distance $d_{\mathbb{L}_{m}}(\boldsymbol{a}, \boldsymbol{c})$ can be defined as the length of any of the shortest maximal chain between $\boldsymbol{a}$ and $\boldsymbol{c}$. In fact, we can state the following theorem.

Theorem 3.1. (De Miguel et al. 2018) Let $\left(L, \leq_{L}\right)$ be a complete lattice satisfying 
condition (MFC). It holds that the lattice $\left(\mathbb{L}_{m}, \leq_{\mathbb{L}_{m}}\right)$ also satisfies condition (MFC). Furthermore, for any $[\boldsymbol{a}, \boldsymbol{c}] \in \mathbb{L}_{m}$, it holds that

$$
d_{\mathbb{L}_{m}}(\boldsymbol{a}, \boldsymbol{c})=d_{L}\left(a_{1}, c_{1}\right)+\ldots+d_{L}\left(a_{m}, c_{m}\right) .
$$

Remark 4. As a consequence, the orness measure introduced in Definition 2.4 can be applied to OWA operators defined on $\left(\mathbb{L}_{m}, \leq_{\mathbb{L}_{m}}\right)$. In addition, it is easy to check that a weight vector $\boldsymbol{\alpha}=\left(\boldsymbol{\alpha}_{1}=\left[\alpha_{11}, \ldots, \alpha_{1 m}\right], \ldots, \boldsymbol{\alpha}_{n}=\left[\alpha_{n 1}, \ldots, \alpha_{n m}\right]\right) \in\left(\mathbb{L}_{m}\right)^{n}$ is distributive in the quadruple $\left(\mathbb{L}_{m}, \leq_{\mathbb{L}_{m}}, \mathbb{T}, \mathbb{S}\right)$ if and only if $\alpha_{* i}=\left(\alpha_{1 i}, \ldots, \alpha_{n i}\right)$ is distributive in the quadruple $\left(L, \leq_{L}, T, S\right)$ for any $1 \leq i \leq m$.

The following result holds.

Theorem 3.2. (De Miguel et al. 2018) Let $\left(L, \leq_{L}, T, S\right)$ a quadruple where $\left(L, \leq_{L}\right)$ is a complete lattice satisfying condition $(M F C)$ and let $\boldsymbol{\alpha}=\left(\boldsymbol{\alpha}_{1}, \ldots, \boldsymbol{\alpha}_{n}\right)$ be a distributive weight vector in the quadruple $\left(\mathbb{L}_{m}, \leq_{\mathbb{L}_{m}}, \mathbb{T}, \mathbb{S}\right)$. Then,

$$
\operatorname{orness}_{\mathbb{L}_{m}}\left(F_{\boldsymbol{\alpha}}\right)=\frac{1}{\mu_{1}+\ldots+\mu_{m}}\left(\mu_{1} \operatorname{orness}_{L}\left(F_{\alpha_{* 1}}\right)+\ldots+\mu_{m} \operatorname{orness}_{L}\left(F_{\alpha_{* m}}\right)\right),
$$

where for each $i \in\{1, \ldots, m\}, \alpha_{* i}=\left(\alpha_{1 i}, \ldots, \alpha_{n i}\right)$ denotes the distributive weight vector and $\mu_{i}=\sum_{k=1}^{n} d_{L}\left(Q_{\alpha_{* i}}(k-1), Q_{\alpha_{* i}}(k)\right)$.

We focus now in the lattice $\mathbb{I}_{m}$ comprising all the $m$ dimensional real intervals:

$$
\mathbb{I}_{m}=\left\{\boldsymbol{a}=\left[a_{1}, \ldots, a_{m}\right] \mid 0 \leq a_{1} \leq \ldots \leq a_{m} \leq 1\right\},
$$

with the order relation given by

$$
\boldsymbol{a} \leq \boldsymbol{c} \text { if and only if } a_{i} \leq c_{i} \text { for } i \in\{1, \ldots, m\} .
$$

Different t-norms and t-conorms are considered in different subsections.

Note that the symbol $\leq$ is used here both for denoting the order relation on $I=[0,1]$ and on $\mathbb{I}_{m}$.

Although $\left(\mathbb{I}_{m}, \leq\right)$ is not an MFC-lattice, different distances can be considered on it in order to define a quantitative orness for OWA operators. Taking into account the result shown in Theorem 3.1 we propose the distance between any $m$ dimensional intervals $\boldsymbol{a}, \boldsymbol{c} \in \mathbb{I}_{m}$ given by:

$$
d_{\mathbb{I}_{m}}(\boldsymbol{a}, \boldsymbol{c})=d_{I}\left(a_{1}, c_{1}\right)+\ldots+d_{I}\left(a_{m}, c_{m}\right)=\left|c_{1}-a_{1}\right|+\ldots+\left|c_{m}-a_{m}\right| .
$$

Observe that, in this case, the equality

$$
d_{\mathbb{I}_{m}}(\boldsymbol{a}, \boldsymbol{c})=d_{I}\left(a_{1}, c_{1}\right)+\ldots+d_{I}\left(a_{m}, c_{m}\right)
$$

is not a fact, as in Theorem 3.1, but a definition.

After this choice, a quantitative orness can be defined following the same steps than in Definition 2.4.

Definition 3.3. Let $\left(\mathbb{I}_{m}, \leq\right)$ be the lattice comprising all the $m$-dimensional intervals with bounds in $I=[0,1]$ and let $T$ and $S$ be respectively a t-norm and a t-conorm 
defined on $I$. For any distributive weight vector $\boldsymbol{\alpha}=\left(\boldsymbol{\alpha}_{1}, \ldots, \boldsymbol{\alpha}_{n}\right)$ in $\left(\mathbb{I}_{m}, \leq, \mathbb{T}, \mathbb{S}\right)$, consider the qualitative quantifier $Q_{\alpha}:\{0,1, \ldots, n\} \rightarrow \mathbb{I}_{m}$ :

$$
\begin{aligned}
& Q_{\boldsymbol{\alpha}}(0)=[0, \ldots, 0] \\
& Q_{\boldsymbol{\alpha}}(j)=\mathbb{S}\left(\boldsymbol{\alpha}_{1}, \ldots, \boldsymbol{\alpha}_{j}\right) \text { for } j=1, \ldots, n .
\end{aligned}
$$

For each $j=1, \ldots, n$, we denote $\mu(j)=d_{\mathbb{I}_{m}}\left(Q_{\boldsymbol{\alpha}}(j-1), Q_{\boldsymbol{\alpha}}(j)\right)$ and call $\mu=\mu(1)+$ $\ldots+\mu(n)$.

Now, define

$$
\operatorname{orness}_{\mathbb{I}_{m}}\left(F_{\boldsymbol{\alpha}}\right)=\frac{1}{n-1} \sum_{j=1}^{n}(n-j) \frac{\mu(j)}{\mu} .
$$

Theorem 3.4. Let $\left(\mathbb{I}_{m}, \leq, \mathbb{T}, \mathbb{S}\right)$ denote the quadruple of the lattices comprising all the $m$-dimensional interval with bounds in $[0,1]$ and let $T$ and $S$ be respectively a $t$-norm and a t-conorm in $[0,1]$. If $\alpha_{* i}=\left(\alpha_{1 i}, \ldots, \alpha_{n_{i}}\right) \in I^{n}$ is a distributive weight vector in $(I, \leq, T, S)$ for each $i \in\{1, \ldots, m\}$, then the following results hold:

(1) The value $\mu_{i}=\sum_{k=1}^{n} \mu_{i}(k)=1$ for any $i \in\{1, \ldots, m\}$.

(2) The value $\mu=\sum_{k=1}^{m} \mu(k)=m$.

(3) $\operatorname{orness}_{\mathbb{I}_{m}}\left(F_{\boldsymbol{\alpha}}\right)=\frac{1}{m} \sum_{k=1}^{m} \operatorname{orness}_{I}\left(F_{\alpha_{* k}}\right)$.

Proof. (1) For each $i=1, \ldots, m$, consider the distributive weight vector $\alpha_{* i}=$ $\left(\alpha_{1 i}, \ldots, \alpha_{n i}\right)$ in $(I, \leq, T, S)$. The quantifier $Q_{\alpha_{* i}}:\{0,1, \ldots, n\} \rightarrow I$ is given by $Q_{\alpha_{* i}}(0)=0$ and, for $j=1, \ldots, n, Q_{\alpha_{* i}}(j)=S\left(\alpha_{1 i}, \ldots, \alpha_{j i}\right)$. For simplicity, we denote $Q_{\alpha_{* i}}(j)$ by $q_{j i}$. Hence, it holds that $\mu_{i}(j)=d_{I}\left(Q_{\alpha_{* i}}(j-1), Q_{\alpha_{* i}}(j)\right)=$ $\left(q_{j i}-q_{(j-1) i}\right)$, which is non-negative because $Q_{\alpha_{* i}}$ is monotonically increasing. We find that

$$
\mu_{i}=\mu_{i}(1)+\ldots \mu_{i}(n)=\sum_{i=1}^{n}\left(q_{j i}-q_{(j-1) i}\right)=q_{n i}-q_{0 i}=1 .
$$

(2) On the other hand, since the quantifier $Q_{\alpha}:\{0,1, \ldots, n\} \rightarrow \mathbb{I}_{m}$ is given by

$$
\begin{aligned}
& Q_{\boldsymbol{\alpha}}(0)=[0, \ldots, 0] \\
& Q_{\boldsymbol{\alpha}}(j)=\mathbb{S}\left(\boldsymbol{\alpha}_{1}, \ldots, \boldsymbol{\alpha}_{j}\right)=\left[q_{j 1}, \ldots, q_{j m}\right] \text { for } j=1, \ldots, n,
\end{aligned}
$$

we have, for each $j=1, \ldots, n$,

$$
\mu(j)=d_{\mathbb{I}_{m}}\left(Q_{\boldsymbol{\alpha}}(j-1), Q_{\boldsymbol{\alpha}}(j)\right)=\sum_{i=1}^{m} d_{I}\left(Q_{\alpha_{* i}}(j-1), Q_{\alpha_{* i}}(j)\right)=\sum_{i=1}^{m} \mu_{i}(j) .
$$


Therefore,

$$
\begin{aligned}
& \mu=\sum_{j=1}^{n} \mu(j)=\sum_{j=1}^{n} \sum_{i=1}^{m} \mu_{i}(j)=\sum_{i=1}^{m} \sum_{j=1}^{n} \mu_{i}(j)= \\
& \sum_{i=1}^{m} \mu_{i}=1+\ldots+1=m .
\end{aligned}
$$

(3) Now, orness $\mathbb{I}_{m}\left(F_{\boldsymbol{\alpha}}\right)=$

$$
\begin{aligned}
& \frac{1}{n-1} \sum_{j=1}^{n}(n-j) \frac{\mu(j)}{\mu}=\frac{1}{m(n-1)} \sum_{j=1}^{n}(n-j) \sum_{i=1}^{m} \mu_{i}(j) \\
& =\frac{1}{m(n-1)} \sum_{i=1}^{m}\left(\sum_{j=1}^{n}(n-j) \mu_{i}(j)\right)=\frac{1}{m} \sum_{i=1}^{m}\left(\operatorname{orness}_{I}\left(F_{\alpha_{* i}}\right)\right) .
\end{aligned}
$$

Note that this formula does not involve the t-norm $\mathbb{T}$ chosen, except for the requirement of distributivity of the weight vector. Only the t-conorm $\mathbb{S}$ and the distance considered on the lattice $\mathbb{I}_{m}$ are involved.

Example 3.5. For each $k \in\{1, \ldots, n\}$, we calculate the quantitative orness of the $k$-th statistics $A_{k}:\left(\mathbb{I}_{m}\right)^{n} \rightarrow \mathbb{I}_{m}$, which is the $n$-ary OWA operator determined by the weight vector

$$
\boldsymbol{\alpha}=\left(\boldsymbol{\alpha}_{1}, \ldots, \boldsymbol{\alpha}_{n}\right) \text { with } \boldsymbol{\alpha}_{k}=[1, \ldots, 1] \text { and } \boldsymbol{\alpha}_{j}=[0, \ldots, 0] \text { for any } j \neq k .
$$

Note that $\mathbb{S}\left(\boldsymbol{\alpha}_{1}, \ldots, \boldsymbol{\alpha}_{n}\right)=[1, \ldots, 1]$. In addition, $\boldsymbol{\alpha}$ is always distributive because

$$
\mathbb{T}\left[\boldsymbol{a}, \mathbb{S}\left(\boldsymbol{\alpha}_{1}, \ldots, \boldsymbol{\alpha}_{n}\right)\right]=\boldsymbol{a}=\mathbb{S}\left[\mathbb{T}\left(\boldsymbol{a}, \boldsymbol{\alpha}_{1}\right), \ldots, \mathbb{T}\left(\boldsymbol{a}, \boldsymbol{\alpha}_{n}\right)\right] .
$$

Moreover, in this case,

$$
Q_{\boldsymbol{\alpha}}(j)=\left\{\begin{array}{ll}
{[0, \ldots, 0]} & \text { if } j<k \\
{[1, \ldots, 1]} & \text { otherwise }
\end{array} \text { and } \mu(j)= \begin{cases}m & \text { if } j=k \\
0 & \text { otherwise }\end{cases}\right.
$$

Now, by Theorem 3.4 (ii), we have

$$
\operatorname{orness}\left(F_{\boldsymbol{\alpha}}\right)=\frac{1}{m(n-1)} \sum_{j=1}^{n}(n-j) \mu(j)=\frac{1}{m(n-1)}(n-k) m=\frac{n-k}{n-1} .
$$

\section{1. $\mathbb{I}_{m}$ with the product $t$-norm and the Lukasievicz $t$-conorm}

First, we consider on $(I, \leq)$ the t-norm $T$ given by the product and the Lukasievicz t-conorm $S$. Then the t-norm $\mathbb{T}$ on $\left(\mathbb{I}_{m}, \leq\right)$ is given by

$$
\mathbb{T}(\boldsymbol{a}, \boldsymbol{c})=\left(a_{1} \cdot c_{1}, \ldots, a_{m} \cdot c_{m}\right)
$$


and the t-conorm $\mathbb{S}$ on $\left(\mathbb{I}_{m}, \leq\right)$ is given by

$$
\mathbb{S}(\boldsymbol{a}, \boldsymbol{c})=\left(\min \left\{a_{1}+c_{1}, 1\right\}, \ldots, \min \left\{a_{m}+c_{m}, 1\right\}\right) .
$$

Proposition 3.6. Let $\left(\mathbb{I}_{m}, \leq, \mathbb{T}, \mathbb{S}\right)$ be the quadruple described above. A weight vector $\boldsymbol{\alpha}=\left(\boldsymbol{\alpha}_{1}=\left[\alpha_{11}, \ldots, \alpha_{1 m}\right], \ldots, \boldsymbol{\alpha}_{n}=\left[\alpha_{n 1}, \ldots, \alpha_{n m}\right]\right) \in\left(\mathbb{I}_{m}\right)^{n}$ is distributive in $\left(\mathbb{I}_{m}, \leq\right.$ $, \mathbb{T}, \mathbb{S})$ if and only if the following two conditions hold.

(1) $\alpha_{1 i}+\ldots+\alpha_{n i}=1$, for all $1 \leq i \leq m$.

(2) $\alpha_{11}=\ldots=\alpha_{1 m} ; \alpha_{21}=\ldots=\alpha_{2 m} ; \ldots ; \alpha_{n 1}=\ldots=\alpha_{n m}$.

Proof. By Remark 4, the weight vector $\boldsymbol{\alpha}=\left(\boldsymbol{\alpha}_{1}=\left[\alpha_{11}, \ldots, \alpha_{1 m}\right], \ldots, \boldsymbol{\alpha}_{n}=\right.$ $\left.\left[\alpha_{n 1}, \ldots, \alpha_{n m}\right]\right)$ is distributive in $\left(\mathbb{I}_{m}, \leq, \mathbb{T}, \mathbb{S}\right)$ if and only if, for each $1 \leq i \leq m$, $\alpha_{* i}=\left(\alpha_{1 i}, \ldots, \alpha_{n i}\right)$ is distributive in $(I, \leq, T, S)$.

The weight vector $\alpha_{* i}$ is distributive in $(I, \leq, T, S)$ if $S\left(\alpha_{1 i}, \ldots, \alpha_{n i}\right)=1$ and it holds that $T\left(a, S\left(\alpha_{1 i}, \ldots, \alpha_{n i}\right)\right)=T(a, 1)=S\left(T\left(a, \alpha_{1 i}\right), \ldots, T\left(a, \alpha_{n i}\right)\right)$ for any $a \in I$.

Since we are considering Lukasievicz t-norm and t-conorm, it holds that:

$$
a=T(a, 1)=S\left(a \alpha_{1 i}, \ldots, a \alpha_{n i}\right)=\min \left\{a\left(\alpha_{1 i}+\ldots+\alpha_{n i}\right), 1\right\}, \text { for any } a \in I
$$

which only holds if $\alpha_{1 i}+\ldots+\alpha_{n i}=1$.

But in that case, it must be

$$
\alpha_{11}=\ldots=\alpha_{1 m} ; \alpha_{21}=\ldots=\alpha_{2 m} ; \ldots ; \alpha_{n 1}=\ldots=\alpha_{n m} .
$$

Indeed, since $\boldsymbol{\alpha}_{j} \in \mathbb{I}_{m}$, it means that $\alpha_{j 1} \leq \ldots \leq \alpha_{j m}$ for each $1 \leq j \leq n$. If $\alpha_{j i}<\alpha_{j(i+1)}$ for some $j \in\{1, \ldots, n\}$ and some $i \in\{1, \ldots, m-1\}$, then

$$
1=\alpha_{1 i}+\ldots+\alpha_{j i}+\ldots+\alpha_{n i}<\alpha_{1(i+1)}+\ldots+\alpha_{j(i+1)}+\ldots+\alpha_{n(i+1)}=1,
$$

which is not possible.

Theorem 3.7. Let $\left(\mathbb{I}_{m}, \leq, \mathbb{T}, \mathbb{S}\right)$ be the quadruple described above. For each distributive weight vector $\boldsymbol{\alpha}=\left(\boldsymbol{\alpha}_{1}=\left[\alpha_{1}, \ldots, \alpha_{1}\right], \ldots, \boldsymbol{\alpha}_{n}=\left[\alpha_{n}, \ldots, \alpha_{n}\right]\right)$ in $\left(\mathbb{I}_{m}, \leq, \mathbb{T}, \mathbb{S}\right)$, we have

$$
\operatorname{orness}_{\Pi_{m}}\left(F_{\boldsymbol{\alpha}}\right)=\operatorname{orness}_{I}\left(F_{\alpha}\right) \text {, }
$$

where $\alpha=\left(\alpha_{1}, \ldots, \alpha_{n}\right)$ denotes the distributive weight vector in $(I, \leq, T, S)$.

Proof. Note that, by Proposition 3.6, it is clear that for any $i=1, \ldots, m$, the weight vectors

$$
\alpha_{* 1}=\alpha_{* 2}=\ldots=\alpha_{* n}=\left(\alpha_{1}, \ldots, \alpha_{n}\right),
$$

are distributive in $(I, \leq, T, S)$. Then, by Theorem 3.4 :

$$
\operatorname{orness}_{\mathbb{I}_{m}}\left(F_{\boldsymbol{\alpha}}\right)=\frac{1}{m}\left(\operatorname{orness}_{I}\left(F_{\alpha_{* 1}}\right)+\ldots+\operatorname{orness}_{I}\left(F_{\alpha_{* m}}\right)\right)=\operatorname{orness}_{I}\left(F_{\alpha}\right),
$$

where $\alpha=\left(\alpha_{1}, \ldots, \alpha_{n}\right)$.

Remark 5. Note that the value orness ${ }_{I}\left(F_{\alpha}\right)$ coincides with that defined by Yager. 
We show that, in the particular case of this subsection, in which the considered t-norm and t-conorms are the product and Lukasievicz, respectively, the weight vector can be retrieved from the qualitative quantifier.

Proposition 3.8. Let $\left(\mathbb{I}_{m}, \leq, \mathbb{T}, \mathbb{S}\right)$ be the quadruple described above.

For each monotonically increasing function $Q:\{0,1, \ldots, n\} \rightarrow I$ with $Q(0)=0$ and $Q(n)=1$, there exists a unique distributive weight vector $\boldsymbol{\alpha}$ in $\left(\mathbb{I}_{m}, \leq, \mathbb{T}, \mathbb{S}\right)$ with the qualitative quantifier $Q_{\boldsymbol{\alpha}}$ satisfying

$$
Q_{\boldsymbol{\alpha}}(j)=[Q(j), \ldots, Q(j)] \text { for } 1 \leq j \leq n .
$$

Proof. Define the weight vector $\boldsymbol{\alpha}$ by:

$$
\begin{aligned}
& \boldsymbol{\alpha}_{1}=[Q(1), \ldots, Q(1)] \\
& \boldsymbol{\alpha}_{2}=[Q(2)-Q(1), \ldots, Q(2)-Q(1)] \\
& \vdots \\
& \boldsymbol{\alpha}_{n}=[Q(n)-Q(n-1), \ldots, Q(n)-Q(n-1)]
\end{aligned}
$$

By Definition 2.4, we have

$$
\begin{aligned}
Q_{\boldsymbol{\alpha}}(0)= & {[0, \ldots, 0]=[Q(0), \ldots, Q(0)] } \\
& \quad \text { and, for } j=1, \ldots, n, \\
Q_{\boldsymbol{\alpha}}(j)= & \mathbb{S}\left(\boldsymbol{\alpha}_{1}, \ldots, \boldsymbol{\alpha}_{j}\right)=[Q(1)+(Q(2)-Q(1))+\ldots+(Q(j)-Q(j-1)) \\
& , \ldots, Q(1)+(Q(2)-Q(1))+\ldots+(Q(j)-Q(j-1))] \\
= & {[Q(j), \ldots, Q(j)] }
\end{aligned}
$$

In particular,

$$
Q_{\boldsymbol{\alpha}}(n)=\mathbb{S}\left(\boldsymbol{\alpha}_{1}, \ldots, \boldsymbol{\alpha}_{n}\right)=[Q(n), \ldots, Q(n)]=[1, \ldots, 1],
$$

whence the weight vector $\boldsymbol{\alpha}$ is distributive.

In order to show the unicity, suppose that $\boldsymbol{\beta}$ is a distributive weight vector with $Q_{\boldsymbol{\beta}}(j)=[Q(j), \ldots, Q(j)]$ for $1 \leq j \leq n$. We find that

$$
\begin{aligned}
& \boldsymbol{\beta}_{1}=Q_{\boldsymbol{\beta}}(1)=[Q(1), \ldots, Q(1)]=Q_{\boldsymbol{\alpha}}(1)=\boldsymbol{\alpha}_{1} \text { and }, \text { for any } j=2, \ldots, n, \\
& \boldsymbol{\beta}_{j}=Q_{\boldsymbol{\beta}}(j)-Q_{\boldsymbol{\beta}}(j-1)=[Q(j)-Q(j-1), \ldots, Q(j)-Q(j-1)] \\
& =Q_{\boldsymbol{\alpha}}(j)-Q_{\boldsymbol{\alpha}}(j-1)=\boldsymbol{\alpha}_{j},
\end{aligned}
$$

where the subtraction between $m$-dimensional intervals is made componentwise.

\section{2. $\mathbb{I}_{m}$ with the t-norm given by the meet and the $t$-conorm given by the join}

Consider now the lattice $\mathbb{I}_{m}$ endowed with the t-norm given by the meet $\wedge$ and the t-conorm given by the join $\vee$. In this case, the lattice $\left(\mathbb{I}_{m}, \leq, \wedge, \vee\right)$ is distributive. 
Table 1. Range of the linguistic labels according to the different experts.

\begin{tabular}{|c|c|c|c|c|}
\hline \hline & healthy & unhealthy & very unhealthy & hazardous \\
\hline Expert 1 & {$[0,100]$} & {$[100,200]$} & {$[200,300]$} & {$[300,500]$} \\
\hline Expert 2 & {$[0,150]$} & {$[150,250]$} & {$[250,350]$} & {$[350,500]$} \\
\hline Expert 3 & {$[0,125]$} & {$[125,200]$} & {$[200,350]$} & {$[350,500]$} \\
\hline
\end{tabular}

Proposition 3.9. Let $\boldsymbol{\alpha}=\left(\boldsymbol{\alpha}_{1}=\left[\alpha_{11}, \ldots, \alpha_{1 m}\right], \ldots, \boldsymbol{\alpha}_{n}=\left[\alpha_{n 1}, \ldots, \alpha_{n m}\right]\right) \in\left(\mathbb{I}_{m}\right)^{n}$ be a weight vector in $\left(\mathbb{I}_{m}, \leq, \mathbb{T}, \mathbb{S}\right)$. The vector $\boldsymbol{\alpha}$ is distributive if and only if $\boldsymbol{\alpha}_{j}=$ $[1, \ldots, 1]$ for some $j \in\{1, \ldots, n\}$.

Proof. Note that $\boldsymbol{\alpha}$ is a weight vector if and only if $\boldsymbol{\alpha}_{1} \vee \ldots \vee \boldsymbol{\alpha}_{n}=[1, \ldots, 1]$. This means, in particular, that $\alpha_{11} \vee \ldots \vee \alpha_{n 1}=1$, which holds only if $\alpha_{j 1}=1$ for some $j \in\{1, \ldots, n\}$. Since $\boldsymbol{\alpha}_{j} \in \mathbb{I}_{m}$, then $\boldsymbol{\alpha}_{j}=[1, \ldots, 1]$.

The following result, which was proved in (De Miguel et al. 2018), asserts than it is possible to retrieve the weight vector from the qualitative quantifier in this case.

Theorem 3.10. Let $\left(\mathbb{I}_{m}, \leq, \wedge, \vee\right)$ be the quadruple described above.

For each monotonically increasing function $Q:\{0,1, \ldots, n\} \rightarrow \mathbb{I}_{m}$ with $Q(0)=$ $[0, \ldots, 0]$ and $Q(n)=[1, \ldots, 1]$, it holds that

(1) There exists some weight vector $\boldsymbol{\alpha}$ in $\left(\mathbb{I}_{m}, \leq, \mathbb{T}, \vee\right)$ with $Q_{\boldsymbol{\alpha}}(j)=Q(j)$ for any $1 \leq j \leq n$.

(2) Such a weight vector $\boldsymbol{\alpha}$ is not necessarily unique. However, if both $\boldsymbol{\alpha}$ and $\boldsymbol{\beta}$ are weight vectors in $\left(\mathbb{I}_{m}, \leq, \wedge, \vee\right)$ with $Q_{\boldsymbol{\alpha}}=Q_{\boldsymbol{\beta}}$, then the OWA operators $F_{\boldsymbol{\alpha}}$ and $F_{\boldsymbol{\beta}}$ agree in $\mathbb{I}_{m}$.

\section{An application of interval-valued OWA operators in order to find a good partition}

The Department of Environment is studying the air pollution of a certain region. For this purpose, they need to classify the air quality taking into account four different linguistic labels: healthy, unhealthy, very unhealthy and hazardous. They ask prestigious experts about the appropriate range of the different linguistic labels but their answers do not coincide as it is stated in Table 1.

In order to choose the best partition of the ranges and to achieve a consensus between the three experts, the Department of Environment has carried out an independent study to fuse the different ranges into a single one which represents the consensus of all the opinions.

First of all, each expert opinion is mathematically represented as a 3-dimensional interval considering the three bounds in which the linguistics labels change according to each expert. Taking into account that the Air Quality Index (AQI) may vary between 0 and 500, these values are normalized.

Expert 1: $[100,200,300]$ normalized to $[0.2,0.4,0.6]$

Expert 2: $[150,250,350]$ normalized to $[0.3,0.5,0.7]$

Expert 3: $[125,200,350]$ normalized to $[0.25,0.4,0.7]$ 
Table 2. Partition generated aggregating with the weight vector $\boldsymbol{\alpha}$.

\begin{tabular}{|c|c|c|c|c|}
\hline & healthy & unhealthy & very unhealthy & hazardous \\
\hline Partition 1 & {$[0,140]$} & {$[140,230]$} & {$[230,350]$} & {$[350,500]$} \\
\hline
\end{tabular}

Table 3. Partition generated aggregating with the weight vector $\boldsymbol{\beta}$.

\begin{tabular}{|c|c|c|c|c|}
\hline & healthy & unhealthy & very unhealthy & hazardous \\
\hline Partition 2 & {$[0,120]$} & {$[120,210]$} & {$[210,330]$} & {$[330,500]$} \\
\hline
\end{tabular}

The final ranges of each linguistic label are computed aggregating the preceding 3 -intervals. In this case, the aggregation process is carried out by means of an OWA operator $F_{\boldsymbol{\alpha}}$ considering different weight vectors.

For the computation of the OWA operators the performing of $b_{1}, b_{2}$ and $b_{3}$ is required.

$$
\begin{aligned}
\boldsymbol{b}_{1} & =[0.2,0.4,0.6] \vee[0.3,0.5,0.7] \vee[0.25,0.4,0.7]=[0.3,0.5,0.7] \\
\boldsymbol{b}_{2} & =[0.2,0.4,0.6] \vee[0.2,0.4,0.6] \vee[0.25,0.4,0.7]=[0.25,0.4,0.7] \\
\boldsymbol{b}_{3}=[0.2,0.4,0.6] \wedge[0.3,0.5,0.7] \wedge[0.25,0.4,0.7] & =[0.2,0.4,0.6]
\end{aligned}
$$

\subsection{Aggregation by means of $O W A$ operators on $\left(\mathbb{I}_{m}, \leq, \mathbb{T}, \mathbb{S}\right)$}

For fusing the different ranges in $\left(\mathbb{I}_{m}, \leq, \mathbb{T}, \mathbb{S}\right)$, where $\mathbb{T}$ is the $t$-norm given by the product and $\mathbb{S}$ is the Lukasievicz t-conorm, the following distributive weighting vectors are considered:

$$
\begin{aligned}
\boldsymbol{\alpha} & =([0.6,0.6,0.6],[0.4,0.4,0.4],[0,0,0]) \text { and } \\
\boldsymbol{\beta} & =([0.2,0.2,0.2],[0.4,0.4,0.4],[0.4,0.4,0.4]) .
\end{aligned}
$$

The result of the aggregation process by means of $F_{\boldsymbol{\alpha}}$ is

$$
\begin{aligned}
& F_{\boldsymbol{\alpha}}([0.2,0.4,0.6],[0.3,0.5,0.7],[0.25,0.4,0.7])= \\
& {[0.6,0.6,0.6] \cdot[0.3,0.5,0.7]+[0.4,0.4,0.4] \cdot[0.25,0.4,0.7]} \\
& +[0,0,0] \cdot[0.2,0.4,0.6]=[0.18,0.3,0.42]+[0.1,0.16,0.28]+[0,0,0] \\
& =[0.28,0.46,0.7]
\end{aligned}
$$

which generates the partition included in Table 2 .

Similarly, by means of $F_{\boldsymbol{\beta}}$ the result of the aggregation process is

$$
\begin{aligned}
& F_{\boldsymbol{\beta}}([0.2,0.4,0.6],[0.3,0.5,0.7],[0.25,0.4,0.7])= \\
& {[0.2,0.2,0.2] \cdot[0.3,0.5,0.7]+[0.4,0.4,0.4] \cdot[0.25,0.4,0.7]} \\
& +[0.4,0.4,0.4] \cdot[0.2,0.4,0.6]=[0.06,0.1,0.14]+[0.1,0.16,0.28] \\
& +[0.08,0.16,0.24]=[0.24,0.42,0.66]
\end{aligned}
$$

which generates the partition included in Table 3.

The influence of the chosen weighting vector can be quantified by means of the quantitative orness of the OWA operators $F_{\boldsymbol{\alpha}}$ and $F_{\boldsymbol{\beta}}$, which are obtained by means of the quantitative orness of the following OWA operators defined on $(I, \leq, T, S)$ : 
Table 4. Partition generated aggregating with the weight vector $\gamma$.

\begin{tabular}{|c|c|c|c|c|}
\hline \hline & healthy & unhealthy & very unhealthy & hazardous \\
\hline Partition 3 & {$[0,150]$} & {$[150,250]$} & {$[250,350]$} & {$[350,500]$} \\
\hline
\end{tabular}

(1) $F_{\alpha}$ with $\alpha=(0.6,0.4,0) \in I^{3}$.

The quantifier $Q_{\alpha}:\{0,1,2,3\} \rightarrow I$ is given by

$Q_{\alpha}(0)=0, Q_{\alpha}(1)=0.6, Q_{\alpha}(2)=Q_{\alpha}(3)=1$,

whence $\mu(1)=0.6, \mu(2)=0.4, \mu(3)=0$.

Therefore $\operatorname{orness}_{I}\left(F_{\alpha}\right)=$

$$
\frac{1}{n-1} \sum_{k=1}^{3}(n-k) \mu(k)=\frac{1}{2}(2 \cdot 0.6+1 \cdot 0.4)=0.8
$$

(2) $F_{\beta}$ with $\beta=(0.2,0.4,0.4) \in I^{3}$.

The quantifier $Q_{\beta}:\{0,1,2,3\} \rightarrow I$ is given by $Q_{\beta}(0)=0, Q_{\beta}(1)=0.2, Q_{\beta}(2)=0.6 ; Q_{\beta}(3)=1$, whence $\mu(1)=0.2 ; \mu(2)=\mu(3)=0.4$

Therefore $\operatorname{orness}_{I}\left(F_{\beta}\right)=$

$$
\frac{1}{n-1} \sum_{k=1}^{3}(n-k) \mu(k)=\frac{1}{2}(2 \cdot 0.2+1 \cdot 0.4)=0.4
$$

Now, Theorem 3.7 gives:

$$
\begin{aligned}
& \operatorname{ornesS}_{I_{3}}\left(F_{\boldsymbol{\alpha}}\right)=\operatorname{orness}_{I}\left(F_{\alpha}\right)=0.8 \text { and } \\
& \operatorname{ornesS}_{\mathbb{I}_{3}}\left(F_{\boldsymbol{\beta}}\right)=\operatorname{orness}_{I}\left(F_{\beta}\right)=0.4
\end{aligned}
$$

\subsection{Aggregation by means of $O W A$ operators on $\left(\mathbb{I}_{m}, \leq, \wedge, \vee\right)$}

For fusing the different ranges, the lattice $\left(\mathbb{I}_{m}, \leq, \wedge, \vee\right)$ where $\mathbb{T}$ is the t-norm $\wedge$ and $\mathbb{S}$ is the t-conorm $\vee$ is also considered. In this lattice the considered weighting vectors are:

$$
\begin{aligned}
\boldsymbol{\gamma} & =([1,1,1],[0,0.4,1],[0,0,0.5]) \text { and } \\
\boldsymbol{\delta} & =([0,0.2,0.3],[0.2,0.4,0.5],[1,1,1])
\end{aligned}
$$

The result of the aggregation process by means of $F_{\gamma}$ is

$$
\begin{aligned}
& F_{\boldsymbol{\alpha}}([0.2,0.4,0.6],[0.3,0.5,0.7],[0.25,0.4,0.7])= \\
& ([1,1,1] \wedge[0.3,0.5,0.7]) \vee([0,0.4,1] \wedge[0.25,0.4,0.7]) \\
& \vee([0,0,0.5] \wedge[0.2,0.4,0.6])= \\
& {[0.3,0.5,0.7] \vee[0,0.4,0.7] \vee[0,0,0.5]=[0.3,0.5,0.7],}
\end{aligned}
$$

which generates the partition included in Table 4. 
Table 5. Partition generated aggregating with the weight vector $\boldsymbol{\delta}$.

\begin{tabular}{|c|c|c|c|c|}
\hline & healthy & unhealthy & very unhealthy & hazardous \\
\hline Partition 4 & {$[0,100]$} & {$[100,200]$} & {$[200,300]$} & {$[300,500]$} \\
\hline
\end{tabular}

The result of the aggregation process by means of $F_{\boldsymbol{\delta}}$ is:

$$
\begin{aligned}
& F_{\delta}([0.2,0.4,0.6],[0.3,0.5,0.7],[0.25,0.4,0.7])= \\
& ([0,0.2,0.3] \wedge[0.3,0.5,0.7]) \vee([0.2,0.4,0.5] \wedge[0.25,0.4,0.7]) \\
& \vee([1,1,1] \wedge[0.2,0.4,0.6]) \\
& =[0,0.2,0.3] \vee[0.2,0.4,0.5] \vee[0.2,0.4,0.6]=[0.2,0.4,0.6]
\end{aligned}
$$

which generates the partition included in Table 5 .

The influence of the chosen weighting vector can be quantified by means of the quantitative orness of the OWA operators $F_{\boldsymbol{\gamma}}$ and $F_{\boldsymbol{\delta}}$, which are obtained by means of the quantitative orness of the following OWA operators defined on $(I, \leq, \wedge, \vee)$ :

(1) $F_{\gamma_{i}}$ with $\gamma_{1}=(1,0,0), \gamma_{2}=(1,0.4,0), \gamma_{3}=(1,1,0.5)$ in $I^{3}$ :

For $i=1,2,3$, the quantifier $Q_{\gamma_{i}}:\{0,1,2,3\} \rightarrow I$ is given by $Q_{\gamma_{i}}(0)=0, Q_{\gamma_{i}}(1)=Q_{\gamma_{i}}(2)=Q_{\gamma_{i}}(3)=1$, whence $\mu_{i}(1)=1, \mu_{i}(2)=\mu_{i}(3)=0$.

Therefore $\operatorname{orness}_{I}\left(F_{\gamma_{i}}\right)=\frac{1}{2}(2 \cdot 1)=1$

(2) $F_{\delta_{1}}$ with $\delta_{1}=(0,0.2,1) \in I^{3}$ :

The quantifier $Q_{\delta_{1}}:\{0,1,2,3\} \rightarrow I$ is given by $Q_{\delta_{1}}(0)=0, Q_{\delta_{1}}(1)=0, Q_{\delta_{1}}(2)=0.2, Q_{\delta_{1}}(3)=1$, whence $\mu_{1}(1)=0 ; \mu_{1}(2)=0.2 ; \mu_{1}(3)=0.8$

Therefore $\operatorname{orness}_{I}\left(F_{\delta_{1}}\right)=\frac{1}{2}(2 \cdot 0+1 \cdot 0.2)=0.1$

(3) $F_{\delta_{2}}$ with $\delta_{2}=(0.2,0.4,1) \in I^{3}$ :

The quantifier $Q_{\delta_{2}}:\{0,1,2,3\} \rightarrow I$ is given by $Q_{\delta_{2}}(0)=0, Q_{\delta_{2}}(1)=0.2, Q_{\delta_{2}}(2)=0.4, Q_{\delta_{2}}(3)=1$, whence $\mu_{2}(1)=\mu_{2}(2)=0.2 ; \mu_{2}(3)=0.6$

Therefore $\operatorname{orness}_{I}\left(F_{\delta_{2}}\right)=\frac{1}{2}(2 \cdot 0.2+1 \cdot 0.2)=0.3$

(4) $F_{\delta_{3}}$ with $\delta_{3}=(0.3,0.5,1) \in I^{3}$ :

The quantifier $Q_{\delta_{3}}:\{0,1,2,3\} \rightarrow I$ is given by $Q_{\delta_{3}}(0)=0, Q_{\delta_{3}}(1)=0.3, Q_{\delta_{3}}(2)=0.5, Q_{\delta_{3}}(3)=1$, whence $\mu_{3}(1)=0.3 ; \mu_{3}(2)=0.2 ; \mu_{3}(3)=0.5$

Therefore $\operatorname{orness}_{I}\left(F_{\delta_{3}}\right)=\frac{1}{2}(2 \cdot 0.3+1 \cdot 0.2)=0.4$ 
Table 6. Summary of the aggregation processes associated with different weights. OWA operators $F_{\boldsymbol{\alpha}}$ and $F_{\boldsymbol{\beta}}$ are considered on $\left(\mathbb{I}_{3}, \leq, \mathbb{T}, \mathbb{S}\right)$, while $F_{\boldsymbol{\gamma}}$ and $F_{\boldsymbol{\delta}}$ are considered on $\left(\mathbb{I}_{3}, \leq, \wedge, \vee\right)$

\begin{tabular}{|c|c|l|l|}
\hline & $\begin{array}{c}\text { Quantitative } \\
\text { orness } \\
\text { of each OWA } \\
\text { operator }\end{array}$ & $\begin{array}{l}\text { Aggregation } \\
\text { result of opinions } \\
\text { by means } \\
\text { of each OWA }\end{array}$ & $\begin{array}{l}\text { Bounds of } \\
\text { the considered } \\
\text { range }[0,500]\end{array}$ \\
\hline$\delta$ & $0.2 \hat{6}$ & {$[0.2,0.4,0.6]$} & {$[100,200,300]$} \\
\hline$\beta$ & 0.4 & {$[0.24,0.42,0.66]$} & {$[120,210,330]$} \\
\hline$\alpha$ & 0.8 & {$[0.28,0.46,0.7]$} & {$[140,230,350]$} \\
\hline$\gamma$ & 1 & {$[0.3,0.5,0.7]$} & {$[150,250,350]$} \\
\hline
\end{tabular}

Table 7. Final partition of the considered linguistic labels

\begin{tabular}{|c|c|c|c|}
\hline Level 0 & Level 1 & Level 2 & Level 3 \\
\hline healthy & unhealthy & very unhealthy & hazardous \\
\hline$[0,100]$ & {$[100,200]$} & {$[200,300]$} & {$[300,500]$} \\
\hline
\end{tabular}

Now, Theorem 3.7 gives:

$$
\begin{aligned}
& \operatorname{orness}_{\mathbb{I}_{3}}\left(F_{\gamma}\right)=\frac{1}{3}\left(\operatorname{orness}_{I}\left(F_{\gamma_{1}}\right)+\operatorname{orness}_{I}\left(F_{\gamma_{2}}\right)+\operatorname{orness}_{I}\left(F_{\gamma_{3}}\right)\right)=1 \text { and } \\
& \operatorname{orness}_{\mathbb{I}_{3}}\left(F_{\delta}\right)=\frac{1}{3}\left(\operatorname{orness}_{I}\left(F_{\delta_{1}}\right)+\operatorname{orness}_{I}\left(F_{\delta_{2}}\right)+\operatorname{orness}_{I}\left(F_{\delta_{3}}\right)\right) \\
& =\frac{1}{3}(0.1+0.3+0.4)=0.2 \hat{6}
\end{aligned}
$$

Table 6 summarizes the study of the four possible weighting vectors associated with the OWA operator.

Note that, as expected, the higher the orness is, the higher the aggregated bounds are. This means that the defined orness shows a good behavior and it is a suitable function to measure the distance between an OWA operator and the OR-operator. Hence, a possible election of the best of the four partitions can be done by considering the orness measure.

For instance, in the considered example of the Air Quality, a conservative decision may be taken in order to protect the environment. This means that a weighting vector with a low orness may be more appropriated. For instance, if they choose the weighting vector $\boldsymbol{\delta}$ for the aggregation of the expert opinions, the result is the one included in Table 7. As a consequence, corrective measures will be activated at levels as low as possible.

\section{Conclusions}

In this paper we introduce a quantitative orness measure defined for OWA operators with values on the lattice of all the $m$-dimensional intervals with bounds between 0 and 1 endowed with a t-norm $T$ and a t-conorm $S$. We also show an illustrative example in which the behavior of the orness function is coherent with the aggregated values. This examples deals with a one-dimensional bounded range which should be discretized into 4 different intervals. We consider the lattice of $m$-intervals as an appropriate model for studying the problem of finding a good partition of any continuous and bounded range. 
Hence, the lattice of $m$-dimensional intervals can be a suitable tool in many problems, specially those related with discretization in which a partition (some finite number of bounds) should be considered. The advantage of $m$-intervals is that this model does not require difficult computations. However, further theoretical developments may be convenient. These developments as well as an application in data mining with real data are left to future research.

\section{Funding}

This work has been partially supported by MINECO, AEI/FEDER,UE under grant TIN2016-77356-P and by the Public University of Navarre under the project PJUPNA1.

\section{Notes on contributors}

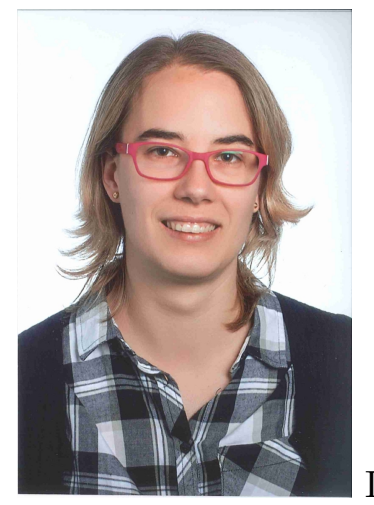

Laura De Miguel received degree in Mathematics from the University of Zaragoza, Spain, in 2012 and the Ph.D. degree in Computer Sciences from the Public University of Navarre, Pamplona, Spain, 2017. She is currently an Assistant Professor in the Department of Statistics, Computer Science and Mathematics at the Public University of Navarre. Her research interests are fuzzy sets and their generalizations, aggregation functions and fuzzy measures.

email: laura.demiguel@unavarra.es

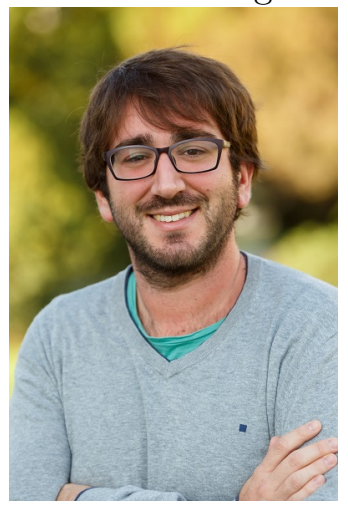

Daniel Paternain received the M.Sc. and Ph.D.degrees in Computer Science from the Public University of Navarre, Pamplona, Spain, in 2008 and2013, respectively. He is currently Lecturer with the Department of Statistics, Computer Science and Mathematics, Public University of Navarre. He is the author and co-author of almost 25 paper in JCR and more than 50 international conference communications. His research interests include both theoretical and applied aspects of 
aggregation functions, image processing and machine learning. His main contributions are based on the use of aggregation techniques and new information fusion procedures, such as aggregation functions, penalty functions or fuzzy integrals in image processing and supervised classification algorithms.

email: daniel.paternain@unavarra.es

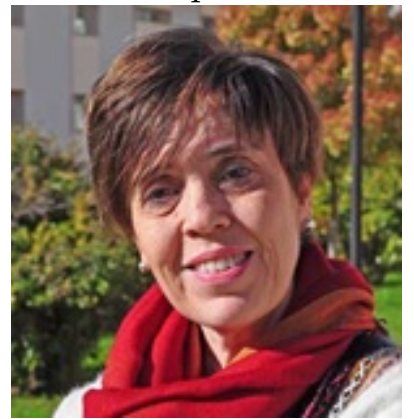

Inmaculada Lizasoain received the Ph.D. degree in mathematics from the Public University of Navarre, Spain, in 1995, where she is currently an Associate Professor with the Department of Statistics, Computer Sciences and Mathematics and INAMAT Research Institute. Her research interests, that include representation group theory, aggregation functions of lattice fuzzy sets and their applications to algebraic automata, decision making and image processing, have given place to more than 20 research papers. email:ilizasoain@unavarra.es

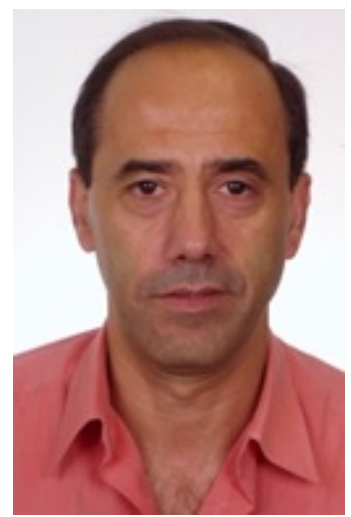

Gustavo Ochoa received the M.Sc.and Ph.D. degrees in mathematics from the University of Zaragoza, Spain, respectively in 1981 and 1984. He is currently an Assistant Lecturer with the Department of Statistic, Computer Science and Mathematics, Public University of Navarre, Pamplona, Spain. His research interests include group theory, representations theory and more recently fuzzy sets and aggregation functions applied to decision making and image processing.

email: ochoa@unavarra.es

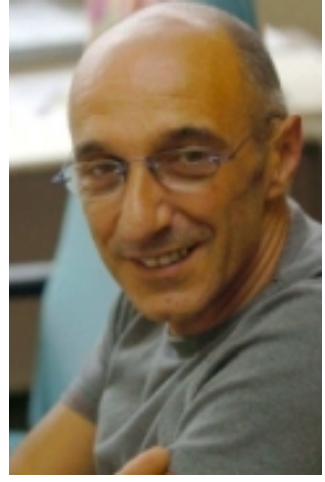

Humberto Bustince is full professor of Computer Science and Artificial Intelligence in the Public University of Navarre. He is the main researcher 
of the Artificial Intelligence and Approximate Reasoning group of this University, whose main research lines are both theoretical (aggregation functions, information and comparison measures, fuzzy sets and extensions) and applied (image processing, classification,machine learning, data mining and big data). He has authored more than 200 works, according to Web of Science, in conferences and international journals, with around 100 of them in journals of the first quartile of JCR. Moreover, five of these works are also among the highly cited papers of the last ten years, according to Science Essential Indicators of Web of Science. He is editor-in-chief of the online magazine Mathware \& Soft Computing of the European Society for Fuzzy Logic and Technologies (EUSFLAT) and of the Axioms journal. He is associated editor of the IEEE Transactions on Fuzzy Systems journal and member of the editorial board of the journals Fuzzy Sets and Systems, Information Fusion, International Journal of Computational Intelligence Systems and Journal of Intelligent \&Fuzzy Systems. He is co-author of a monography about averaging functions and co-editor of several books. He has organized some re-known international conferences such as EUROFUSE 2009 and AGOP 2013. He is Senior member of the IEEE Association and Fellow of the International Fuzzy Systems Association(IFSA).

email: bustince@unavarra.es

\section{References}

Astorino, A., E. Gorgone, M. Gaudioso, and D. Pallaschke. 2011. "Data preprocessing in semisupervised SVM classification." Optimization 60 (1-2): 143-151.

Birkhoff, Garrett. 1967. Lattice Theory. 3rd ed., Vol. 25 of American Mathematical Society Colloquium publications. American Mathematical Society.

Bustince, Humberto, Mikel Galar, Benjamin Bedregal, Anna Kolesarova, and Radko Mesiar. 2013. "A new approach to interval-valued Choquet integrals and the problem of ordering in interval-valued fuzzy set applications." IEEE Transactions on Fuzzy systems 21 (6): 11501162.

Choi, Yoon-Seok, and Byung-Ro Moon. 2004. "Genetic Fuzzy Discretization for Classification Problems." In Genetic and Evolutionary Computation Conference, 1303-1304. Springer.

Davey, B. A., and H. A. Priestley. 1990. Introduction to Lattices and Order. Cambridge University Press.

De Baets, B., and R. Mesiar. 1999. "Triangular norms on product lattices." Fuzzy Sets and Systems 104 (1): 61-75.

De Miguel, L, D Paternain, I Lizasoain, G Ochoa, and H Bustince. 2018. "Orness measurements for lattice m-dimensional interval-valued OWA operators." Applied Mathematics and Computation 339: 63-80.

De Miguel, L., M. Sesma-Sara, M. Elkano, M. Asiain, and H. Bustince. 2017. "An algorithm for group decision making using n-dimensional fuzzy sets, admissible orders and OWA operators." Information Fusion 37: 126 - 131.

De Miguel, Laura, Humberto Bustince, Barbara Pekala, Urszula Bentkowska, Ivanosca Da Silva, Benjamin Bedregal, Radko Mesiar, and Gustavo Ochoa. 2016. "Interval-valued Atanassov intuitionistic OWA aggregations using admissible linear orders and their application to decision making." IEEE Transactions on Fuzzy Systems 24 (6): 1586-1597.

García, Salvador, Julián Luengo, and Francisco Herrera. 2015. Data preprocessing in data mining. Springer.

Jin, L., R. Mesiar, and R. Yager. 2019. "Ordered Weighted Averaging Aggregation on Convex Poset." IEEE Transactions on Fuzzy Systems 27: 612-617.

Komorníková, Magda, and Radko Mesiar. 2011. "Aggregation functions on bounded partially ordered sets and their classification." Fuzzy Sets and Systems 175 (1): 48-56. 
Lavangnananda, K., and S. Chattanachot. 2017. "Study of discretization methods in classification." In 2017 9th International Conference on Knowledge and Smart Technology (KST), Feb, 50-55.

Liu, H., F. Hussain, C. Lim Tan, and M. Dash. 2002. "Discretization: An Enabling Techinque." Data Mining and Knowledge Discovery .

Lizasoain, Inmaculada, and Cristina Moreno. 2013. "OWA operators defined on complete lattices." Fuzzy Sets and Systems 224: 36-52.

Merigó, Jos M., and Montserrat Casanovas. 2010. "The fuzzy generalized OWA operator and its application in strategic decision making." Cybernetics and Systems 41 (5): 359-370.

Mesiar, R., F. Kouchakinejad, A. ipoov, and M. Mashinchi. 2018. "OWA Operators on Complete Lattices." IEEE Transactions on Fuzzy Systems 26 (6): 3884-3887.

Ochoa, G., I. Lizasoain, D. Paternain, H. Bustince, and N. R. Pal. 2017. "From quantitative to qualitative orness for lattice OWA operators." International Journal of General Systems 46 (6): 640-669.

Paternain, D., L. De Miguel, G. Ochoa, I. Lizasoain, R. Mesiar, and H. Bustince. 2019. "The Interval-Valued Choquet Integral Based on Admissible Permutations." IEEE Transactions on Fuzzy Systems 27 (8): 1638-1647.

Paternain, D., G. Ochoa, I. Lizasoain, H. Bustince, and R. Mesiar. 2016. "Quantitative orness for lattice OWA operators." Information Fusion 30: 27 - 35.

Wang, Hai, and Zeshui Xu. 2016. "Total orders of extended hesitant fuzzy linguistic term sets: Definitions, generations and applications." Knowledge-Based Systems 107: 142 - 154.

Wei, Guiwu, Fuad E. Alsaadi, Tasawar Hayat, and Ahmed Alsaedi. 2016. "Hesitant Fuzzy Linguistic Arithmetic Aggregation Operators in Multiple Attribute Decision Making." Iranian Journal of Fuzzy Systems 13 (4): 1-16.

Yager, R. R. 1988. "On ordered weighted averaging aggregation operators in multicriteria decisionmaking." IEEE Transactions on Systems, Man, and Cybernetics 18 (1): 183-190.

Yager, Ronald R. 1993. "Families of OWA operators." Fuzzy Sets and Systems 59 (2): 125 148.

Yager, Ronald R. 2009. "OWA aggregation of intuitionistic fuzzy sets." International Journal of General Systems 38 (6): 617-641. 